\title{
Comment on "Comparison of CT and PET/CT for biopsy guidance in oncological patients"
}

\author{
Long Chen ${ }^{1} \cdot$ Hua Sun ${ }^{1}$
}

Received: 29 June 2017 / Accepted: 3 August 2017 /Published online: 14 August 2017

(C) Springer-Verlag GmbH Germany 2017

Dear editors,

Professor Juliano J. Cerci et al. published their article entitled "Comparison of CT and PET/CT for biopsy guidance in oncological patients" in the European Journal of Nuclear Medicine and Molecular Imaging on February 14, 2017 (doi $10.1007 / \mathrm{s} 00259-017-3658-8$ ). They concluded that there were no significant differences in the ability to obtain a diagnostic specimen or in the complication rates between $\mathrm{PET} / \mathrm{CT}$ and $\mathrm{CT}$ guidance.

We can not agree with the opinions mentioned above. Firstly, they should not have compared the overall diagnosis efficacy among different lesions. It is obvious that both PET/ $\mathrm{CT}$ and $\mathrm{CT}$ harbored their own special specificity and sensitivity to different diseases, so arbitrary comparison between the overall efficacy does not mean too much. They should have focused on some special lesions, for example, the lymphoma, rather make no choice. If we compare the advantage of PET/CT and $\mathrm{X}$ rays in detecting osteosarcoma, $\mathrm{X}$ rays will be excellent. Secondly, CT-guided biopsy is based on the definite lesions, which depends mostly on the contrast enhanced
CT rather than $\mathrm{CT}$ itself, while no contrast is introduced in $\mathrm{PET} / \mathrm{CT}$ in most situations. Consequently, they should not have attributed the high specificity or sensitivity caused by contrast to CT equipment, merely. Finally, when it comes to liver lesions, especially in primary hepatocellular carcinoma (HCC), CT holds an obvious advantage by the dynamic contrast enhancement technique, with an accuracy of $100 \%$, approximately; however, HCC always shows as non FDG-avid. In the current research, 21 patients suffering from liver lesions (accounting for $11.2 \%$ ) went through PET/CT guided biopsy, while 30 (accounting for 20.5\%) went through CT-guided biopsies, which may have sharply increased both the sensitivity and specificity of CT-guided biopsies.

\section{Compliance with ethical standards}

Ethical approval This article does not contain any studies with human participants performed by any of the authors.

Hua Sun

649790827@qq.com

Long Chen

lonechen1983@hotmail.com

1 Department of PET/CT Center, Yunnan Tumor Hospital, The Third Affiliated Hospital of Kunming Medical University, No. 519

Kunzhou Road, Xishan District, Kunming 650118, Yunnan, People's

Republic of China 